\title{
Dynamically Responsive Multifunctional Asymmetric Triblock Terpolymer Membranes with Intrinsic Binding Sites for Covalent Molecule Attachement
}

Qi Zhang, ${ }^{\dagger}$ Yibei Gu, ${ }^{\dagger}$ Yuk Mun Li, ${ }^{\ddagger}$ Peter A. Beaucage, ${ }^{\dagger}$ Teresa Kao, ${ }^{\dagger}$ and Ulrich Wiesner $^{*}$

${ }^{\dagger}$ Department of Materials Science and Engineering and ${ }^{\ddagger}$ Robert Frederick Smith School of Chemical and Biomolecular Engineering, Cornell University, Ithaca, New York 14850, United States

(a)

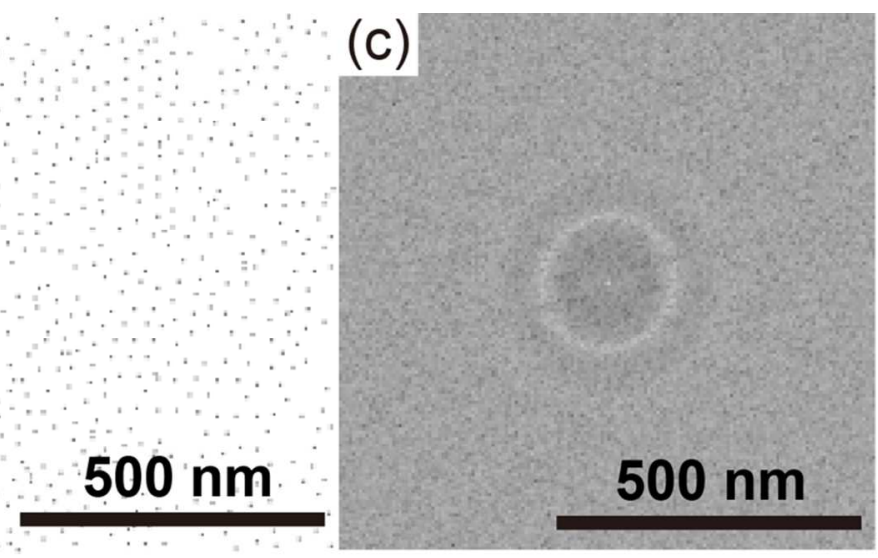

Figure S1. Image processing details for FFT radical profile with ImageJ. (a) Set threshold between o - 22.08 \% for Figure 3a. (b) Find edges and find maxima of Figure S1(a). (c) Fast Fourier Transform of Figure S1(b). Radical profile of FFT image is in Figure $3(\mathrm{~d})$. 
(a)
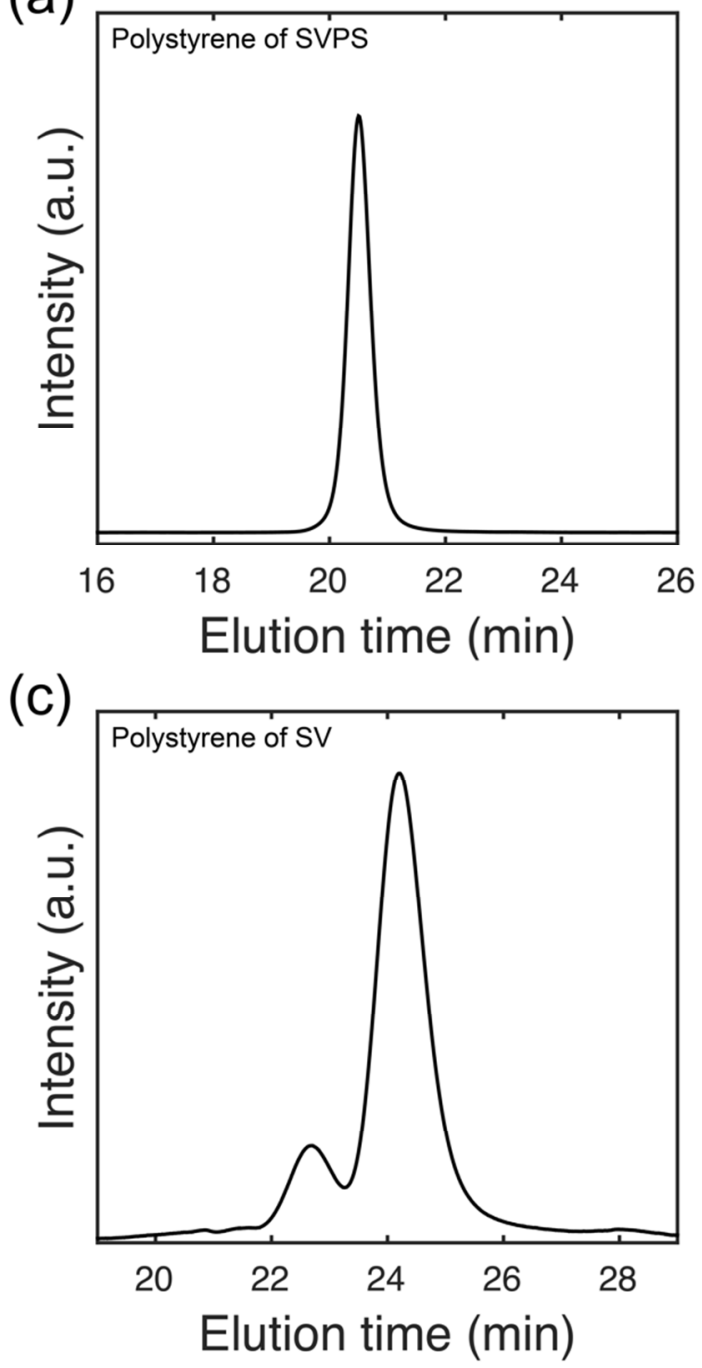

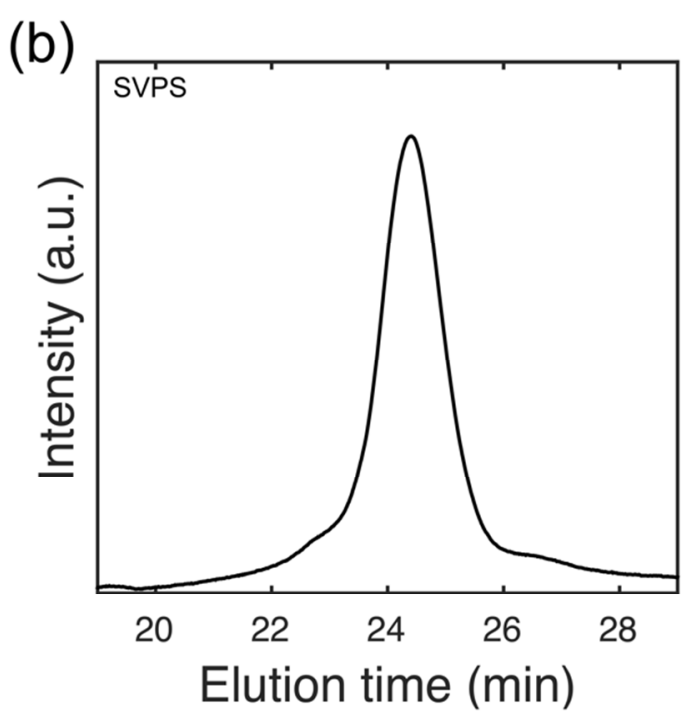

(d)

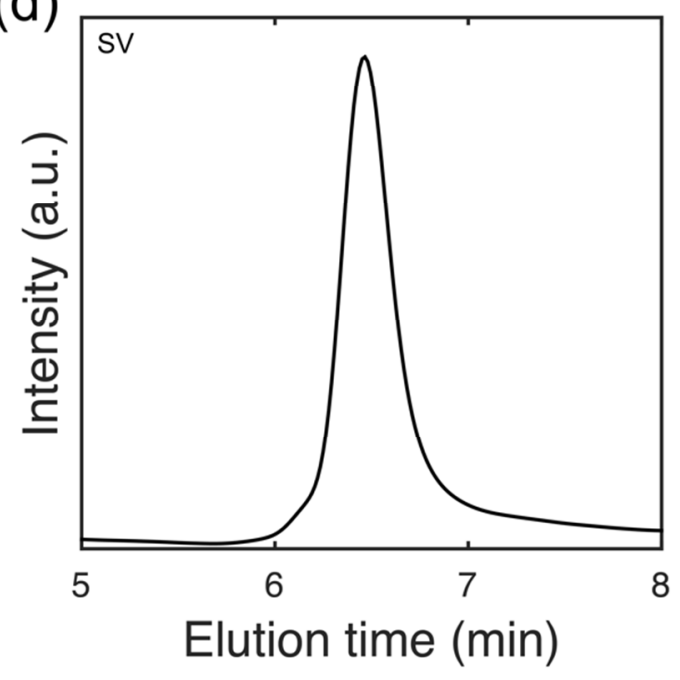

Figure S2. GPC elution curves of SVPS and SV and their polymer intermediates. (a) Polystyrene of SVPS; The GPC measurement was performed in THF on a Waters ambient-temperature GPC system equipped with a Waters 2410 differential refractive index (RI) detector. (b) SVPS; The GPC measurement was performed in DMF as described in the experimental section. (c) Polystyrene of SV; The GPC measurement was performed in DMF as described in the experimental section. The smaller peak on the left at twice the molar mass of the main peak resulted from dimerization of polystyrene. As the GPC curve of the final SV in (d) showed a monomodal distribution, the dimer peak probably resulted from aliquot sampling for GPC via introduction of oxygen and did not represent the mass distribution of the living polystyrene used for further polymerization with poly(4-vinylpyridine). (d) SV. The GPC measurement was performed in THF on an Agilent 1050 instrument equipped with an Agilent PLgel $5 \mu \mathrm{m}$ Mixed-C column and an Agilent 1100 differential refractive index (RI) detector. 


\section{Supporting Information}

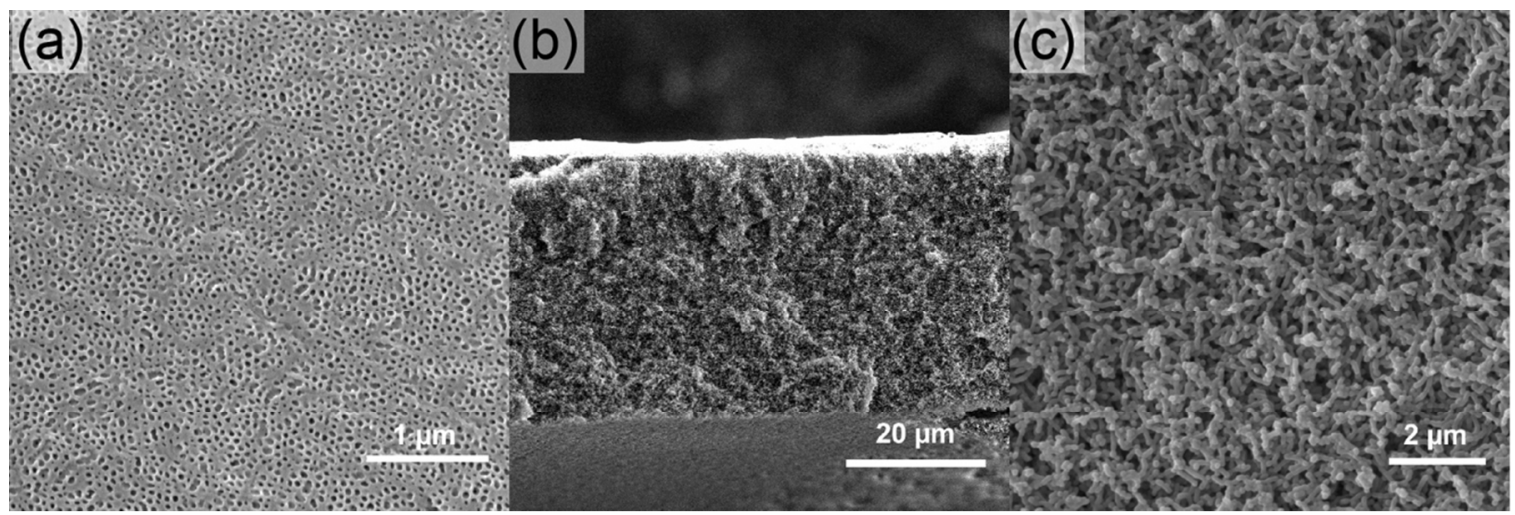

Figure S3. SEM characterization of (a) top surface, (b) cross section, and (c) bottom surface of a SV membrane

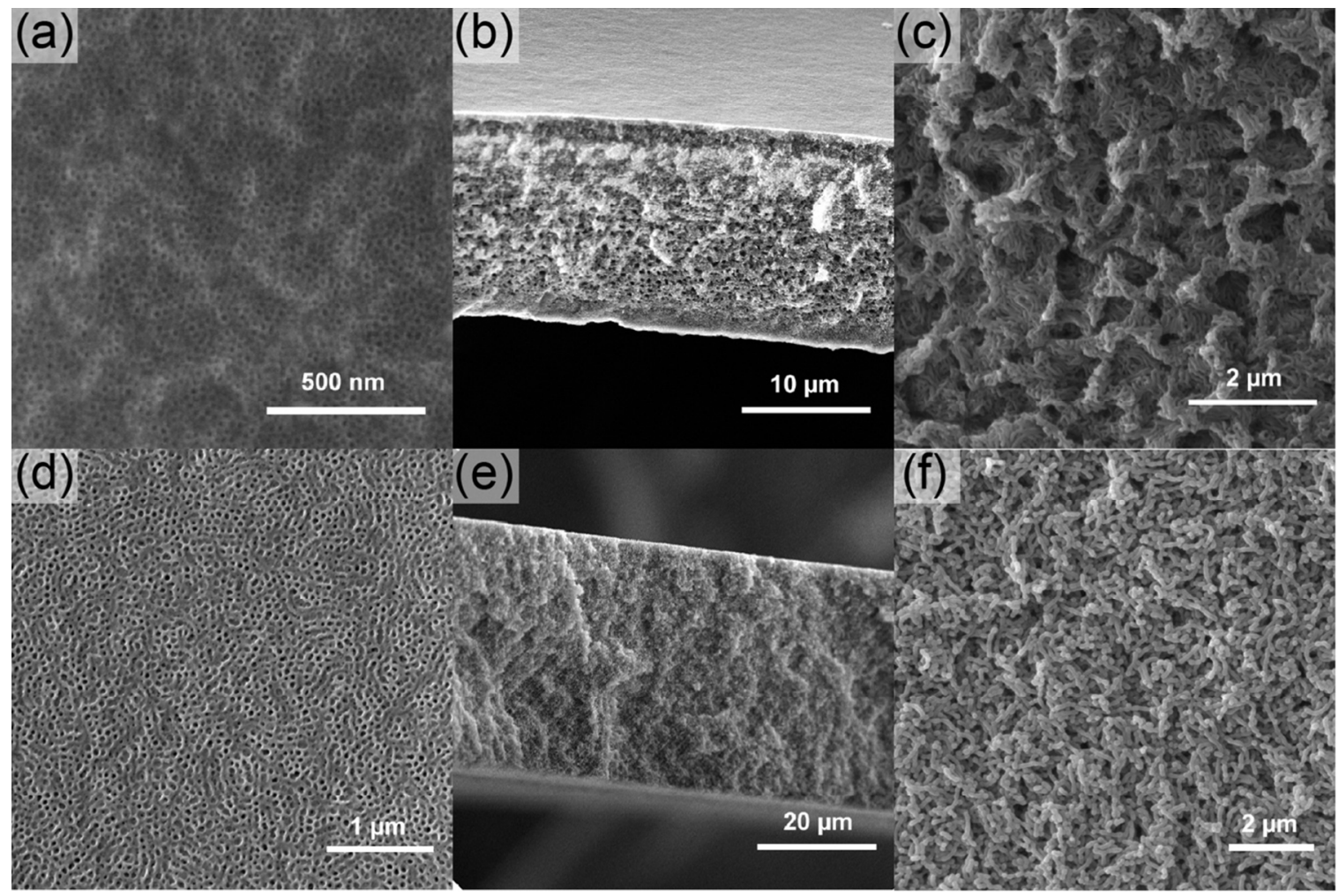

Figure S4. SEM characterization of (a,d) top surfaces, (b,e) cross sections, and (c,f) bottom surfaces of SVPS (a-c) and SV (d-f) membranes washed with DMSO. 